\title{
Wall slip and the nonlinear dynamics of large amplitude oscillatory shear flows
}

\author{
Michael D. Graham \\ Department of Chemical Engineering and Rheology Research Center, \\ University of Wisconsin-Madison, Madison, Wisconsin 53706-1691
}

(Received 5 December 1994; accepted 4 March 1995)

\begin{abstract}
Synopsis
Large amplitude oscillatory shear flows of polymer melts between parallel plates may exhibit complicated nonperiodic responses characteristic of quasiperiodicity or chaos. This complex time dependence is related to the wall slip exhibited by these materials. We use simple models for the fluid elasticity and slip to theoretically and computationally study the nonlinear dynamics of melts in oscillatory shear. The results indicate that both fluid elasticity and a dynamic (e.g. memory-slip) model for the wall slip are necessary for nonperiodic dynamics to occur. Furthermore, when elasticity and a dynamic slip model are coupled, many qualitative features of the dynamics observed in the experiments can be reproduced. In particular, asymmetric periodic responses exhibiting even harmonics are found, as well as quasiperiodic and chaotic motions. Particularly interesting is the prediction of multiple stable periodic motions for a given set of parameters, depending on initial conditions. As a special case, the model reduces to the classical Duffing equation of nonlinear vibration theory. The existence of complex dynamics is robust with respect to changes in both the constitutive model chosen and the details of the wall slip model. (C) 1995 Society of Rheology.
\end{abstract}

\section{INTRODUCTION}

It has long been recognized that polymer melts and other complex fluids may violate the classical no-slip boundary condition at solid surfaces under conditions of high shear stress. Instabilities and complex time dependence are often associated with wall slip in flows of polymer melts, especially extrusion, so it is important to consider the dynamics of the slip process (Denn 1990). In this paper, the interaction between the dynamics of slip and fluid elasticity is studied in the context of large amplitude oscillatory shear flow (LAOS). The analysis is motivated by recent observations of complex time-dependent behavior in experiments on large amplitude oscillatory shear of high density polyethylene (HDPE) (Hatzikiriakos and Dealy 1991), polyurethane (Adrian and Giacomin 1992) and linear low density polyethylene (LLDPE) (Adrian and Giacomin 1994) in sliding plate rheometers. We find that a simple model of the flow and slip dynamics reproduces the qualitative dynamical behavior observed in the experiments and predicts further nonlinear behavior that has not yet been observed experimentally.

The classical experimental example of the effect of slip (or apparent slip) on the stability of a viscoelastic flow is the "spurt" phenomenon (see e.g. Vinogradov et al. 1972). In this experiment, the flow rate through a capillary is observed to jump dramatically at a critical value of the shear stress, a result that may be attributable to the onset of wall slip. Similar instabilities, as well as more complex time-dependent flows ("melt fracture") are observed in extrusion (cf. reviews by Petrie and Denn (1976), Denn 


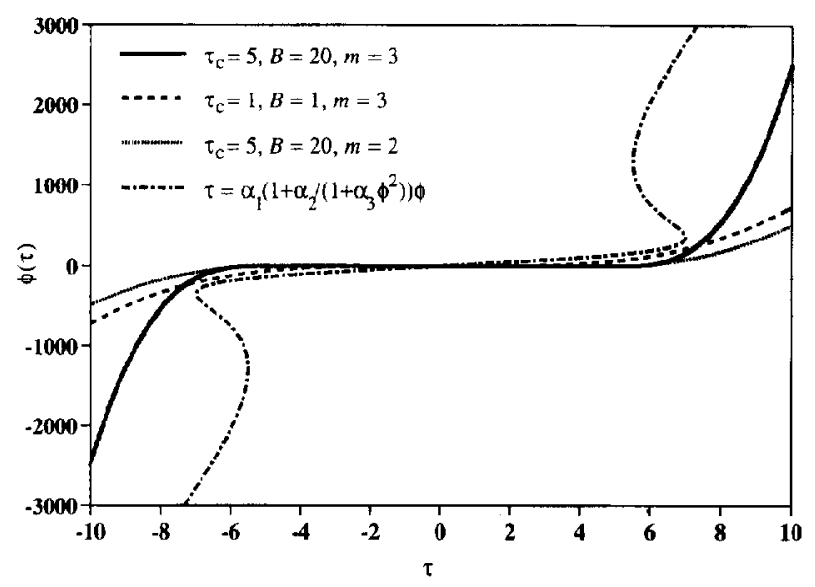

FIG. 1. Various functional forms for the slip velocity function $\phi(\tau)$ in Eq. (7). (a) $\tau_{c}=5, B=20, m=3$, the form used in most of the simulations. (b) $\tau_{c}=1, B=1, m=3$. (c) $\tau_{c}=5, B=20, m=2$, a form used to test the robustness of the model results with respect to the degree of nonlinearity of $\phi$. (d) An example of a multivalued relation (from Georgiou and Crochet 1994): $\tau=\alpha_{1}\left(1+\alpha_{2} /\left(1+\alpha_{3} \phi^{2}\right)\right) \phi$. Here $\alpha_{1}=2 \times 10^{-3}, \alpha_{2}=20, \alpha_{3}=1 \times 10^{-5}$.

(1990), and Larson (1992)). In the general extrusion problem, however, instabilities arise from several mechanisms that may or may not involve slip. Atwood and Schowalter (1989) use a hot-film probe to study the onset of slip for a flow of HDPE in a slit die. The results indicate that the slip velocity oscillates and that these oscillations correspond directly to the observation of regular patterns of extrudate distortion. Lim and Schowalter (1989) make similar observations for polybutadienes, conjecturing that slip and reattachment occur via a relaxation process. In large amplitude oscillatory shear flow of HDPE, Hatzikiriakos and Dealy (1991, hereafter cited as HD) find that the stress response deviates significantly from sinusoidal behavior once the strain amplitude is large enough so that the critical stress for slip is exceeded. In particular, the response is characterized by multiple peaks in stress per cycle. In related experiments on polyurethane, Adrian and Giacomin (1992, hereafter cited as AG) observe periodic responses exhibiting even harmonics, as well as a nonperiodic response that appears quasiperiodic or chaotic. Similar results are observed in LLDPE (Adrian and Giacomin 1994). The presence of even harmonics indicates the occurrence of a symmetry-breaking bifurcation, as the response is no longer commensurate with the symmetry of the experimental geometry and forcing function. AG conjecture that slip is a contributor to the complex dynamics, but do not rule out the possibility that sufficiently nonlinear viscoelasticity is responsible. These LAOS experiments are the motivation for the present work.

The slip phenomenon is often empirically modeled as an algebraic relationship between the slip velocity $u_{s}$ (difference between fluid velocity and wall velocity) and the shear stress $\tau$ :

$$
u_{s}=\phi(\tau)
$$

or more generally,

$$
g\left(\tau, u_{s}\right)=0 .
$$

Figure 1 shows some examples of steady state slip models. The latter form is often used 
because it is observed that for some polymers, the same shear stress can lead to two different stable slip velocities (see Fig. 1d). The multivalued models used to describe these observations exhibit multiple steady states in pressure-driven flow, and if compressibility is taken into account, pulsatile flow (Hatzikiriados and Dealy 1992, Georgiou and Crochet 1994). The present work, however, will consider only models that predict a unique steady state slip velocity for a given shear stress.

An important limitation of these models is that they assume that the slip velocity adjusts instantaneously to the shear stress at the wall, an assumption that may not be valid. Because of this, Pearson and Petrie (1968) introduced a "memory slip model," which in differential form is

$$
u_{s}+\lambda_{s} \dot{u}_{s}=\phi(\tau)
$$

This model assumes that the slip velocity is a functional of the history of the shear stress, with a relaxation time of $\lambda_{s}$ (in dimensionless terms, a "slip Deborah number," $\mathrm{De}_{s}$ ). In contrast to the algebraic or static slip models, Eqs. (1) and (2), the memory-slip model is a dynamic model for the slip and reattachment process. HD proposed that a model like this, in conjunction with a Maxwell model for fluid viscoelasticity, might provide insight into the behavior observed in their experiments, but made no systematic study. A model similar to Eq. (3), but for fluidity rather than slip velocity, has also been studied in the context of oscillatory shear by Yoshimura and Prud'homme (1988). Renardy (1990) shows that the simple shear flow of an upper convected Maxwell fluid with Eq. (3) as a boundary condition is unstable to perturbations of arbitrarily short wavelength. That is, the problem is ill-posed. However, the addition of any finite solvent viscosity to the model renders it well-posed. Nonetheless, the analysis shows that memory-slip behavior may lead to short wave instabilities reminiscent of the sharkskin instability observed during extrusion of melts.

It is also possible for certain constitutive models to display an effective slip phenomenon (a "constitutive instability") even when the no-slip boundary condition is valid. In Poiseuille flow, the Johnson-Segalman equation, for example, predicts a nonunique relationship between shear stress and velocity gradient (Malkus et al. 1991). The consequence of this nonuniqueness is the prediction of flows with a very narrow region of high velocity gradient near the wall and a virtually uniform velocity profile in the interior. This phenomenon has been proposed as an explanation for the observed multiplicity of flow rate for a given wall shear stress observed for many polymer melts. This mechanism is independent of the material constituting the walls. Experiments, however, indicate that in general, the onset of slip does depend on the wall composition (Ramamurthy 1986, HD, Denn 1992, Hatzikiriakos et al. 1995). This work indicates that, at least in some cases, slip is a result of adhesive, rather than cohesive failure. In this work, only models that do not exhibit constitutive instabilities will be considered.

The present work examines the interaction between dynamic slip and viscoelasticity with simple models, the goals being to determine (1) whether these models yield the type of behavior obtained by $\mathrm{AG}$; (2) the robustness of the behavior with respect to variations in the model; and (3) whether the models predict other types of behavior not yet observed in experiment. We first present the models to be studied. Most of the results are obtained using a simple Maxwell model coupled with a memory slip model. We begin the discussion of results by showing that both fluid elasticity and dynamic slip are necessary conditions for nonperiodic behavior in LAOS. This can be shown rigorously for the Maxwell and White-Metzner constitutive models by simple phase space arguments. In the Appendix the result is obtained for a general linear viscoelastic fluid. We examine the model incorporating dynamic slip at a variety of parameter values, presenting examples 
of typical behavior. The model qualitatively reproduces all of the variety of behavior observed by HD and AG, as well as exhibiting another characteristic nonlinear phenomenon: multiple stable responses for a given choice of parameters. As a special case, the model reduces to the well-known Duffing equation, which is known to exhibit multiplicity, symmetry-breaking and chaos. Finally, we examine the robustness of the results by studying the effects of changing the functional form of the slip model, introducing shearthinning and adding a fast-relaxing stress mode to the model. The basic results are found to be robust with respect to these changes in the model.

\section{MODELS}

We are interested in the unsteady response of a sample of polymer melt that is placed between two parallel plates, one of which is oscillating in its own plane with frequency $f$. The plates are a distance $h$ apart. Velocities are scaled with $h f$, stresses with $\eta_{0} f$ and time with the oscillation period $1 / f$. Here $\eta_{0}$ is the zero-shear-rate viscosity of the polymer. For $h$ sufficiently small, the velocity gradient within the sample is approximately constant, and the nominal strain and dimensionless strain rate are given simply by:

$$
\begin{gathered}
\gamma_{n}=\gamma_{0} \sin 2 \pi t, \\
\dot{\gamma}_{n}=2 \pi \gamma_{0} \cos 2 \pi t
\end{gathered}
$$

where $\gamma_{0}$ is the shear strain. However, if wall slip occurs, the actual strain and strain rate will differ from the nominal values; in particular the actual (dimensionless) strain rate $\dot{\gamma}$ is given by:

$$
\dot{\gamma}(t)=\dot{\gamma}_{n}(t)-2 u_{s},
$$

where $u_{s}$ is the dimensionless slip velocity at the stationary wall. This expression assumes that the slip behavior at the two plates is identical. The dynamics of the slip phenomenon are not well-understood; we will assume that slip is governed by a simple relaxation process described by the dimensionless version of Eq. (3):

$$
u_{s}+\mathrm{De}_{s} \dot{u}_{s}=\phi(\tau)
$$

where

$$
\mathrm{De}_{s} \equiv \lambda_{s} f
$$

Here $\tau$ is the dimensionless shear stress and $\lambda_{s}$ is a slip relaxation time, so $\mathrm{De}_{s}$ is the "slip Deborah number." Note that as $\mathrm{De}_{s} \rightarrow 0$, this model reduces to the simple algebraic relation

$$
u_{s}=\phi(\tau)
$$

For the magnitude of $u_{s}$ to be independent of the sign of $\tau$, the function $\phi(\tau)$ must be odd. In their study, HD took $\phi \sim \tau^{3}$; we shall examine a more general class of functions, of the form:

$$
\phi(\tau)=\left\{\begin{array}{l}
0, \quad|\tau|<\tau_{c} \\
B \frac{\tau}{|\tau|}\left(|\tau|-\tau_{c}\right)^{m}, \quad|\tau| \geqslant \tau_{c}
\end{array} .\right.
$$

Figures 1a-c show examples from this class. This functional form is motivated by the observation that in steady shear experiments, the no-slip condition seems to be valid until 
the shear stress reaches a critical value $\tau_{c}$, after which the slip velocity roughly follows a power law in $\tau$ (Atwood and Schowalter 1989, Hill et al. 1990, HD). HD find a power law exponent of 3 for HDPE, whereas Hill et al. (1990), studying LLDPE, find a much stronger dependence, $u_{s} \sim \tau^{6}$.

For the evolution of the stress in the fluid, a number of models are used. The simplest and most extensively used here is the upper convected Maxwell model. In simple shear flows, this model predicts that the shear stress satisfies the simple Maxwell equation:

$$
\tau+\text { De } \dot{\tau}=\dot{\gamma}
$$

where $\mathrm{De}=\lambda f$ is the Deborah number and $\lambda$ the elastic relaxation time (Bird et al. 1987). We also consider the two-mode generalization of this model, where

$$
\begin{gathered}
\tau_{1}+\text { De } \dot{\tau}_{1}=\dot{\gamma}, \\
\tau_{2}+\operatorname{De} \Lambda \dot{\tau}_{2}=H \dot{\gamma}, \\
\tau=\tau_{1}+\tau_{2} .
\end{gathered}
$$

Here $\Lambda$ and $H$ are the ratios of the second relaxation time and viscosity, respectively, to the first. If $\Lambda=H=0$, the single-mode model is recovered. To examine the influence of shear thinning, a White-Metzner model is used:

$$
\tau+\operatorname{De} \eta(\dot{\gamma}) \dot{\tau}=\eta(\dot{\gamma}) \dot{\gamma}
$$

where the viscosity $\eta(\dot{\gamma})$, scaled by $\eta_{0}$, is given by the Carreau equation:

$$
\eta(\dot{\gamma})=\left(1+(\operatorname{De} \dot{\gamma})^{2}\right)^{(n-1) / 2} .
$$

The choice of $\phi(\tau)$ and the constitutive model defines a set of ordinary differential equations to be solved for $u_{s}$ and the stresses. To study the evolution of time-periodic solutions as a parameter is varied and to determine bifurcation points, an interactive version of the parameter continuation software package AUTO is used (Doedel 1981, Taylor and Kevrekidis 1990). For dynamic simulation, the dynamical system simulation program SCIGMA is used (Taylor, Jolly and Kevrekidis 1990). SCIGMA uses the ODESSA package to solve initial value problems (Leis and Kramer 1988).

\section{RESULTS AND DISCUSSION}

Let us begin with a qualitative discussion of the properties of the models that are introduced above. In particular, consider the system of equations comprised of the dynamic slip model, Eq. (5) and the Maxwell constitutive model, Eq. (8). Using the relation between the actual and nominal shear rates, Eq. (4), the equations can be written:

$$
\begin{gathered}
\dot{\tau}=\frac{1}{\mathrm{De}}\left(2 \pi \gamma_{0} \cos 2 \pi t-2 u_{s}-\tau\right), \\
\dot{u}_{s}=\frac{1}{\operatorname{De}_{s}}\left(\phi(\tau)-u_{s}\right) .
\end{gathered}
$$

The phase space for this system of equations is three-dimensional, consisting of the two dependent variables $\left(\tau, u_{s}\right)$ and time. This is the minimum dimension for the existence of time-dependent behavior more complicated than simple periodic oscillation, i.e. quasiperiodicity or chaos. If, however, the slip law is replaced by the algebraic relation, Eq. (6), then the slip velocity is no longer a state variable and the phase space is only 
two-dimensional. Therefore, no nonperiodic behavior can occur [by the PoincareBendixson Theorem (Guckenheimer and Holmes 1986)]. Likewise, if the fluid is Newtonian ( $\mathrm{De} \rightarrow 0$ ), the stress is no longer a state variable and the phase space is again only two-dimensional. Furthermore, it is shown in the Appendix that a general linear viscoelastic liquid with a nonlinear algebraic slip boundary condition cannot exhibit nonperiodic behavior in oscillatory shear. Thus, within the context of these models, both fluid viscoelasticity and dynamic wall slip are necessary conditions for a nonperiodic response.

The phase space argument used for the Maxwell model applies to the nonlinear WhiteMetzner model as well. It does not apply, however, to models like the Giesekus model, because even with an algebraic slip model, the shear and normal stresses are coupled and the phase space is four-dimensional. Nevertheless, simulations of the Giesekus model with an algebraic slip model yield only periodic responses. The simulations of Jeyaseelan and Giacomin (1994) with a multimode model based on transient network theory also yield only periodic responses. Thus it seems that the conclusion that dynamic slip is necessary for nonperiodic behavior in LAOS, which has only been proven for very simple constitutive models, is a general rule. While dynamic slip is necessary for nonperiodicity, it is not sufficient, as the results below will show. Slip can occur without leading to instability. Experimental support for this prediction is discussed by Jeyaseelan and Giacomin (1994).

Returning now to the basic model, Eqs. (11) and eliminating $u_{s}$ gives the second order equation:

$$
\mathrm{DeDe}_{s} \ddot{\tau}+\left(\mathrm{De}+\mathrm{De}_{s}\right) \dot{\tau}+\tau+2 \phi(\tau)=\dot{\gamma}_{n}+\mathrm{De}_{s} \ddot{\gamma}_{n} .
$$

For the particular choice $\phi(\tau)=a \tau^{3}$, this equation is equivalent to the forced Duffing equation, which arises in the theory of nonlinear vibrations (Guckenheimer and Holmes 1986). The Duffing equation has been extensively studied, and displays several interesting phenomena that can now be seen to be relevant to the present problem. The first of these is due to the effect of nonlinearity on the resonant response of the system. The nonlinearity distorts the resonance peak of the oscillator, giving rise to parameter regions where either a small amplitude or a large amplitude response can arise, depending on initial conditions. As the forcing amplitude or frequency is changed, the system can be observed to "jump" suddenly from a weak response to a strong one, or vice versa. This jump occurs at a so-called limit point or saddle-node bifurcation. Due to the nonlinearity, other responses are also observed, including symmetry-breaking bifurcations that lead to the presence of even harmonics in the power spectrum of the response, as well as transitions to chaotic response.

Although the choice $\phi(\tau)=a \tau^{3}$ leads to identification with the Duffing equation, a somewhat more realistic form for $\phi(\tau)$ recognizes that until a large value of $|\tau|$ is reached, the no-slip condition applies at steady state. Thus unless otherwise noted, the results presented below are based on Eq. (7), with $\tau_{c}=5, B=20, m=3$. For an experiment using a frequency $f=2.5 \mathrm{~Hz}$ and a polymer melt with $\eta_{0}=0.01 \mathrm{MPa}$ s (a case studied by HD), this corresponds to a critical stress of $0.125 \mathrm{MPa}$.

We begin the discussion of the computational results with a case that yields responses similar to those observed in experiments and simulations by $\mathrm{HD}$. Let $\mathrm{De}=\mathrm{De}_{s}=\mathbf{0 . 1}$, so the elastic and slip relaxation times are both small. Figures $2 a, 2 b$, and $2 c$ show the shear stress as a function of time for these parameters and three strain amplitudes, $\gamma_{0}=0.5,1.5$ and 5 , respectively. The response at $\gamma_{0}=0.5$ is sinusoidal, and the critical stress has not yet been reached. At 1.5, the critical stress is exceeded for part of the cycle and the fluid slips. This is reflected by the vacillations in stress around the critical value 


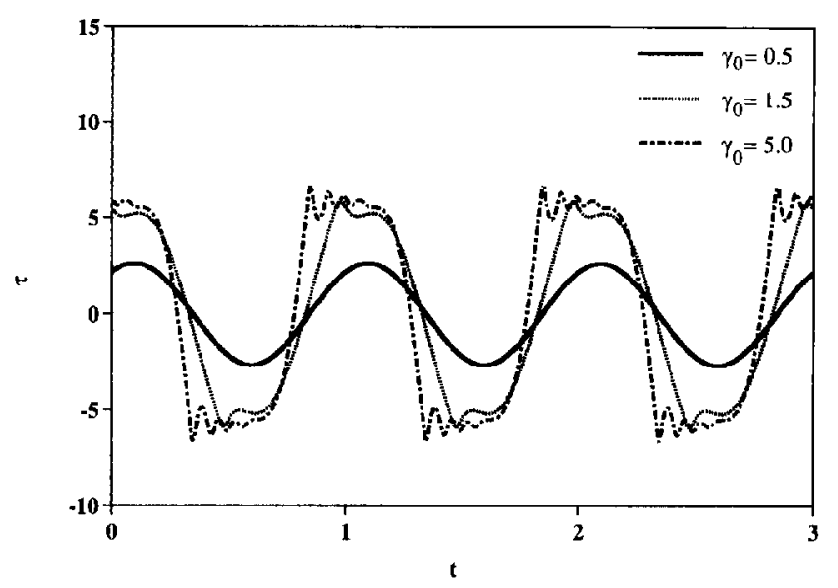

FIG. 2. Periodic responses for $\mathrm{De}=\mathrm{De}_{s}=0.1$. (a) $\gamma_{0}=0.5$; no slip occurs and the response is sinusoidal. (b) $\gamma_{0}=1.5$; the fluid slips slightly, as indicated by the vacillations in the high stress intervals of the response. (c) $\gamma_{0}=5.0$; here the fluid slips and reattaches to the wall several times during each cycle of the wall motion.

during the high stress portions of the cycle. The response changes smoothly from the no-slip to slip regimes as $\gamma_{0}$ increases; there is no bifurcation. At $\gamma_{0}=5$, the effects of slip on the response are much more pronounced than at 1.5. In Fig. 3, the phase space representations of these responses are plotted. Because the fluid is not very elastic, stress relaxation occurs rapidly so there are several cycles of stick/slip alternation (vacillations of the stress response) during each cycle of the wall motion. Strain amplitudes up to 20 were studied; the behavior remains qualitatively similar to that shown here.

If $\mathrm{De}$ and $\mathrm{De}_{s}$ are increased to 1, however, the picture changes dramatically. The overall behavior over a range of nominal strain amplitudes is shown in Fig. 4. We first note that more than one response is observed in some ranges of strain amplitude, depending on initial conditions. Tracing out the solution branch as $\gamma_{0}$ increases (quasistatically)

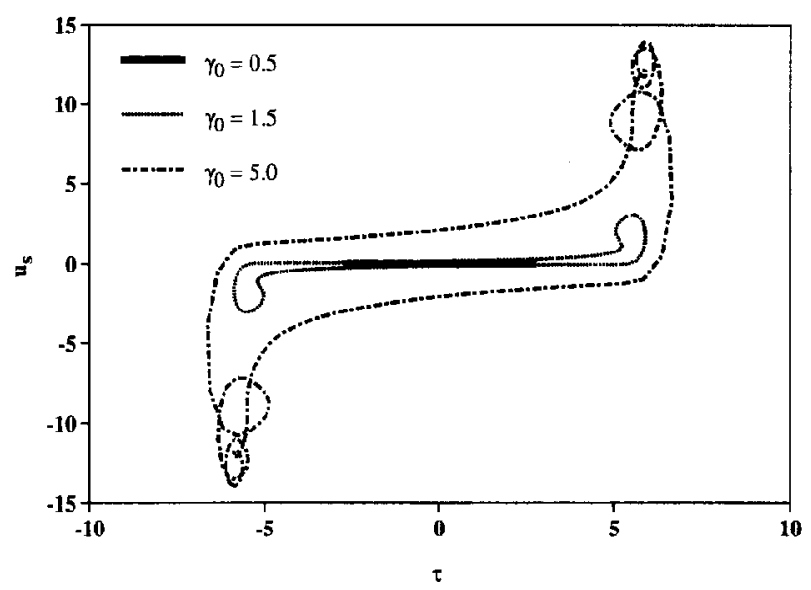

FIG. 3. Phase space representations of the responses shown in Fig. 2. 


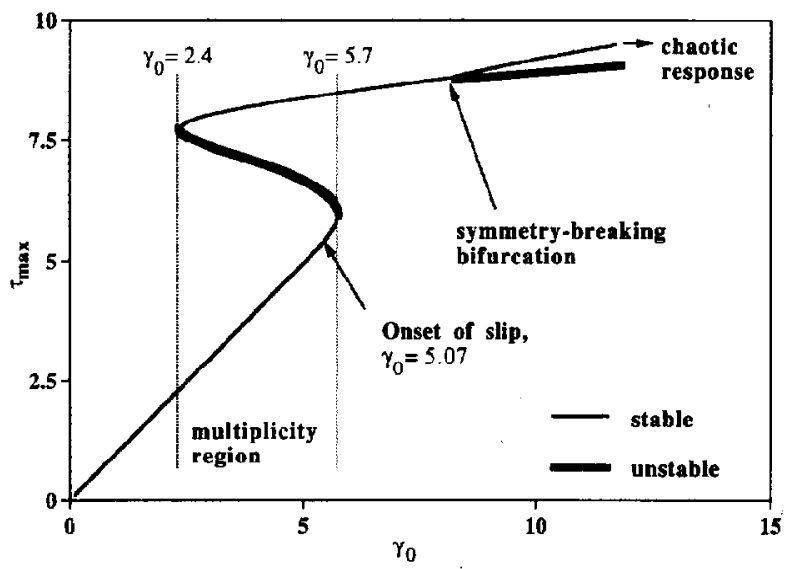

FIG. 4. Bifurcation diagram for the case $\mathrm{De}=\mathrm{De}_{s}=1$. This diagram shows the maximum amplitude of the periodic stress response (vertical axis) as a function of imposed nominal strain amplitude $\gamma_{0}$. In the region $2.4<\gamma_{0}<5.7$, three possible responses exist. The responses with the largest and smallest amplitudes are linearly stable, while the intermediate one is unstable.

reveals that it is S-shaped (i.e. there are two limit point bifurcations) and that multiplicity is present when $\gamma_{0}$ is in the range $2.4-5.7$ (Fig. 4). Whereas this multiplicity of responses is classically found in nonlinear vibrations (and in the Duffing equation), it has yet to be measured in a LAOS experiment. The multiplicity is a purely dynamical effect, as there is only one solution under steady forcing. The lower amplitude branch in Fig. 4 is the expected "no-slip" response. This branch loses existence at the limit point at $\gamma_{0}=5.7$, slightly above the strain amplitude for the onset of slip, which occurs at $\gamma_{0}=5.07$. Figure 5 shows the two stable responses as functions of time when $\gamma_{0}=5$. Here the smaller-amplitude response remains below the threshold of slip, so it is

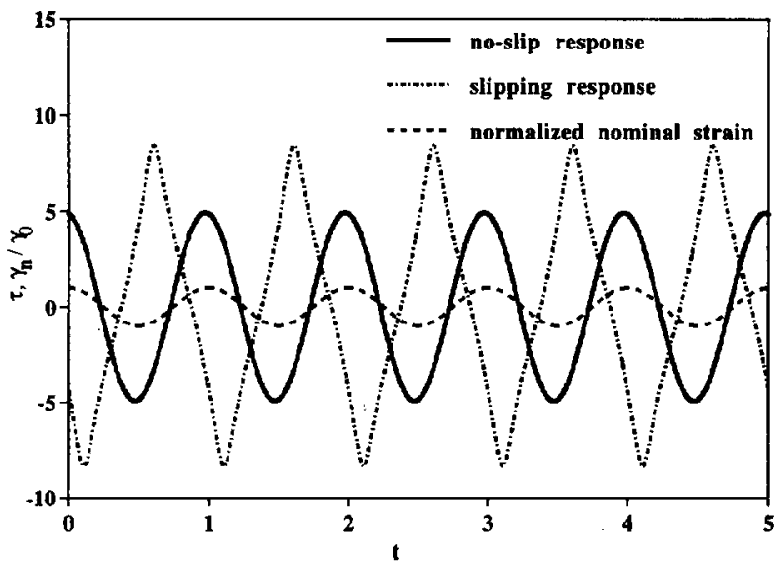

FIG. 5. The stable stress responses when $\mathrm{De}=\mathrm{De} \mathrm{e}_{s}=1, \gamma_{0}=5$. The solid and dot-dashed curves are the responses, while the dashed curve is the nominal strain amplitude normalized by $\gamma_{0}$. 


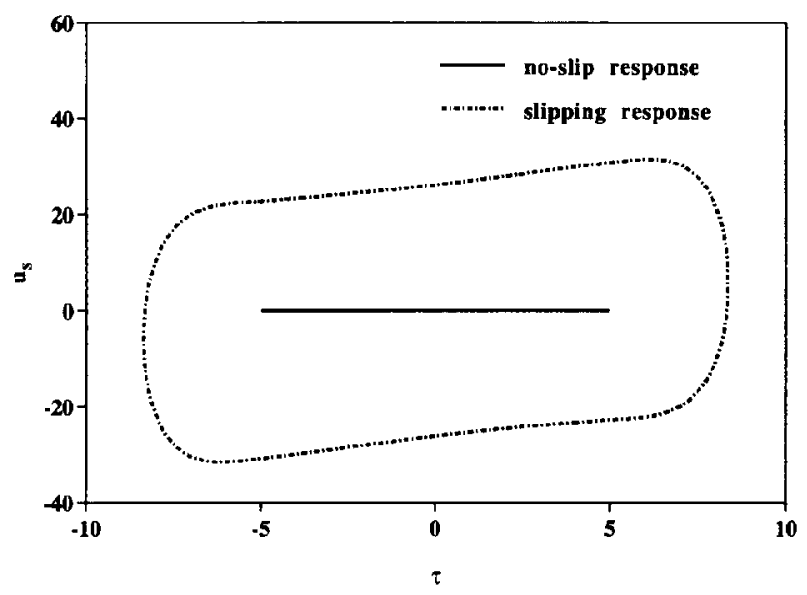

FIG. 6. Phase space representations of the two responses shown in Fig. 5.

sinusoidal, while the larger one exhibits slip. The dashed curve shows the nominal strain amplitude as a function of time. Both stable solutions in Fig. 5 satisfy the symmetry relation

$$
\tau\left(t+\frac{1}{2}\right)=-\tau(t),
$$

indicating that only odd harmonics will appear in the frequency spectrum of the response. Without slip, the response will always obey this symmetry (cf. Appendix). Figure 6 shows the phase space representations of these responses projected onto the $\left(\tau, u_{s}\right)$ plane.

One intuitively expects slip to decrease the stress in a material; at low $\mathrm{De}_{s}$, this expectation is fulfilled. Nevertheless, the above results predict that slip leads to higher stresses. This result arises because the memory slip model incorporates a time lag between the stress and the slip velocity. This lag allows the fluid deformations in a timedependent flow to be larger than they would be without slip.

At slightly higher strains, further interesting behavior appears. Bifurcation analysis with AUTO shows that the periodic solution undergoes a symmetry breaking bifurcation at $\gamma_{0}=7.5$. The stable stress response at $\gamma_{0}=10$ is shown in Fig. 7a. Clearly, the symmetry of Eq. (13) does not describe this response, and even harmonics appear in the frequency spectrum. A similar response is experimentally observed by AG. At higher values of $\gamma_{0}$, a sequence of period-doubling transitions occur, leading to a chaotic response for $\gamma_{0}>14$. The chaotic response at $\gamma_{0}=16$ is shown in Fig. $7 \mathrm{~b}$, and as phase space representations in Fig. 8. The frequency spectrum of this response is shown in Fig. 9, where the presence of even harmonics and high background is clear, as in the experiments of AG. Furthermore, the phase space behavior (in particular the plot of $\dot{\tau}$ versus $\tau$ ) is similar to that observed by AG. As a check on whether the response is actually chaotic, we have computed the correlation dimension of the attractor (Grassberger and Procaccia 1983), finding a value of 2.2. This non-integer dimension indicates that the attractor is fractal, and thus that the response is chaotic. The response remains chaotic until $\gamma_{0} \approx 100$, where the system reverts back to symmetric periodic behavior. AG interpret their experiments as displaying quasiperiodic, not chaotic behavior, hut do not compute any measures of the attractor structure (such as correlation dimension) that would distinguish between chaotic and nonchaotic response. 

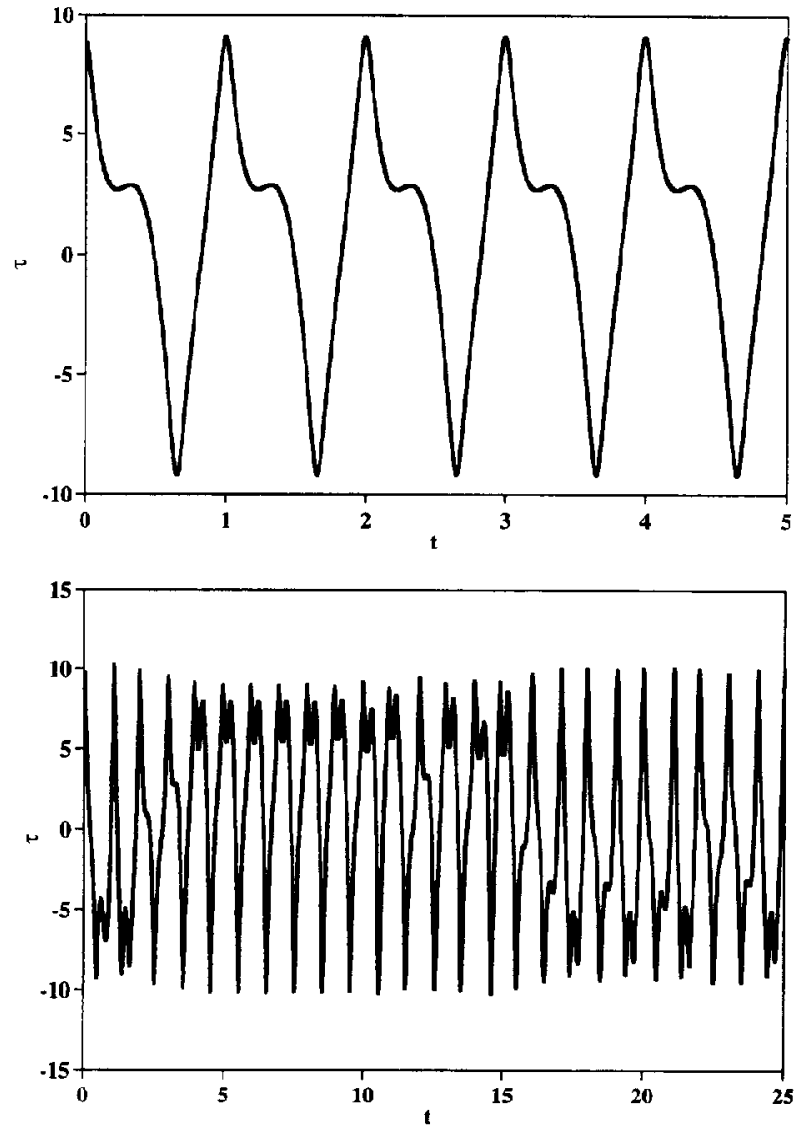

FIG. 7. Stress responses at higher strain amplitudes, $\mathrm{De}=\mathrm{De} e_{s}=1$. (a) $\gamma_{0}=10$; asymmetric, but still periadic sesponse. (b) $\gamma_{0}=16$; chaotic response.

In experiments, quantitative reproducibility of a particular chaotic trajectory is impossible, because of the sensitivity of the trajectory to initial conditions. The overall structure of the attractor, however, is entirely insensitive to small changes in the initial conditions. That is, two trajectories starting from close initial conditions will diverge until the trajectories are no longer correlated; nevertheless, they are moving on the same attractor. This attractor can be characterized by its correlation dimension, as was done here, or by a number of other probabilistic measures. Moon (1992) discusses various methods for diagnosing chaotic responses. Limitations of some of these techniques are discussed by Eckmann and Ruelle (1992), for example.

The response of the model has been computed for a broad range of De,De $e_{s}$ and $\gamma_{0}$. The variety of behavior found when $\mathrm{De}=\mathrm{De}_{s}=1$ and discussed above is typical of the response of the system when strain amplitude and both Deborah numbers are appreciable. Multiplicity is found whenever both De and $\mathrm{De}_{s}$ are greater than about 0.5 , and the range of $\gamma_{0}$ over which multiplicity appears increases as De or $\mathrm{De}_{s}$ increases. Asymmetric behavior is somewhat less pervasive than multiplicity; asymmetric states are found for 

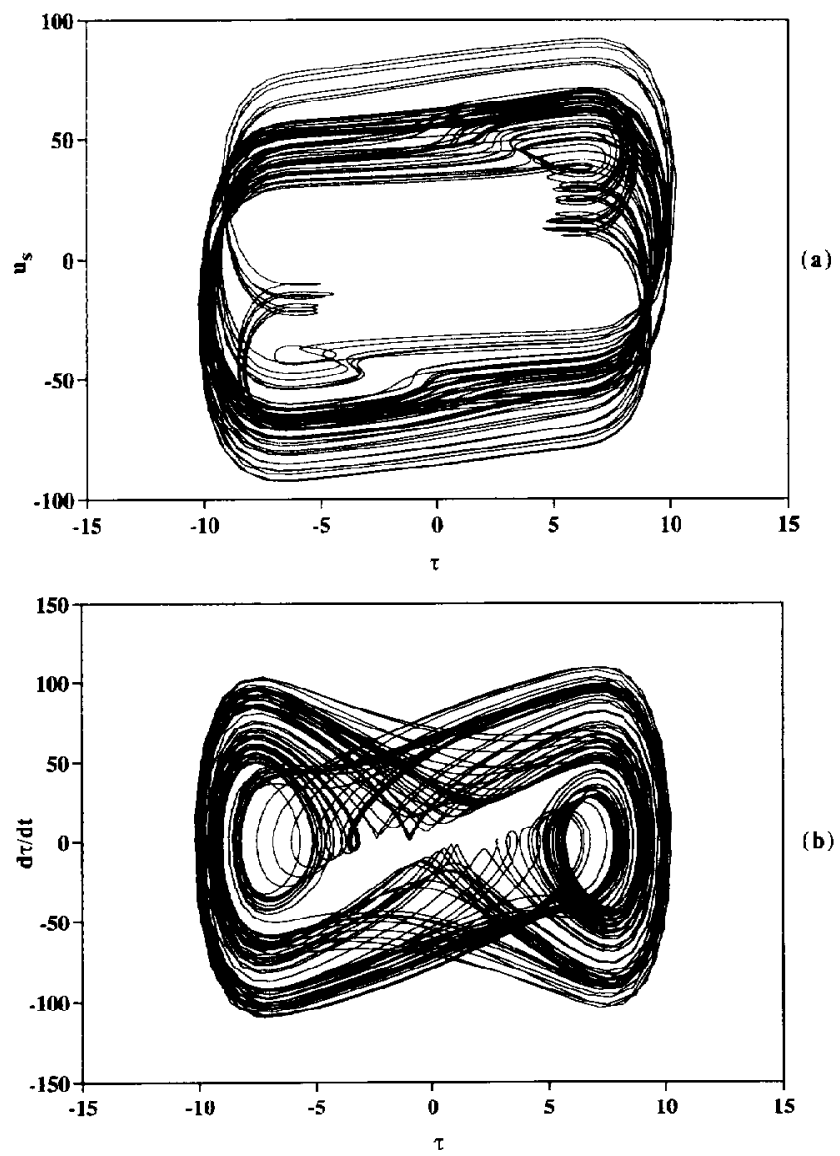

FIG. 8. Phase space representations of the chaotic response shown in Fig. $7 \mathrm{~b}$. (a) $u_{s}$ versus $\tau$, (b) $\tau$ versus $\tau$. The latter is more easily compared with experimental results, because $u_{s}$ is less experimentally accessible.

$\gamma_{0}<20$ only when $\mathrm{De}$ and $\mathrm{De}_{s}$ are between about 0.5 and 2 . If $\mathrm{De} \ll \mathrm{De}_{s}$ or vice versa, only periodic responses are observed; slip may occur, but it does not lead to instability.

The above results have been obtained with a rather simple model. An important issue, then, is whether the results obtained with this model are robust with respect to changes in the model. If the results persist for a variety of model forms, then we can be confident that they are not an artifact of a particular choice for the parameters or functional forms, and therefore that the model provides a solid theoretical framework. We address this issue at several levels. At the simplest level, we retain the form of the model, changing only the parameters $\tau_{c}$ and $B$ in the expression for $\phi(\tau)$, Eq. (5). For $\tau_{c}=B=1$, all the qualitative behavior found above is still present, but at somewhat different values of $\mathrm{De}, \mathrm{De}_{s}$ and $\gamma_{0}$. The same is true if we change $m$ to 2 and use $\tau_{c}=5, B=20$. These two results are important because they indicate that the qualitative results are independent of the details of the nonlinearity.

Another level at which robustness must be addressed is in the choice of constitutive model for the fluid stresses. To study the effect of shear thinning, results have been obtained for the White-Metzner model, Eq. (10), with $\tau_{c}=5, B=20, m=3$, 


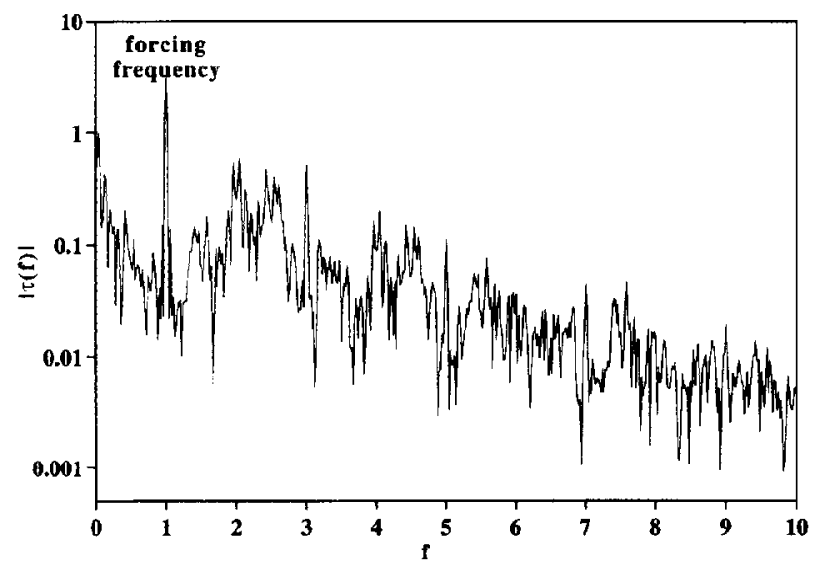

FIG. 9. Frequency spectrum of the chaotic response shown in Figs. 7b and 8 . Note the peaks at the even harmonics and the peak broadening and high baseline characteristic of a chaotic signal.

$\mathrm{De}=\mathrm{De}_{s}=1$, and two different values of $n$. When $n=0.7$ (power law slope of -0.3 ), the results are qualitatively identical to those for the Maxwell model. The multiplicity, symmetry-breaking and chaos are all observed, but shifted to somewhat higher values of $\gamma_{0}$. When $n$ is decreased to 0.6 , the results change somewhat. The multiplicity and symmetry-breaking occur, but only one period-doubling bifurcation appears, instead of the full sequence observed in the previous cases. At $\gamma_{0}=35$, the solution reverts back to a symmetric, periodic response.

We also consider a two-mode Maxwell model, focusing on the case where the second mode has a very rapid response. Thus, in Eq. (9), we fix $\Lambda$ (the ratio of relaxation times) at 0.01 and consider different values of $H$ (the ratio of viscosities). When $H=0.01$, the influence of the second mode is rather weak. The multiplicity region shrinks somewhat and the first symmetry-breaking bifurcation occurs at a slightly higher value, near $\gamma_{0}=11$. This asymmetric branch undergoes a period doubling bifurcation, but at slightly higher values of $\gamma_{0}$ the solution reverts back to a singly periodic solution and finally back to a symmetric response at $\gamma_{0}=23$. This is distinguished from the single mode case, where a sequence of period doubling bifurcations occur, leading to a chaotic response that persists until $\gamma_{0} \approx 100$. If $H$ is increased to 1 , the change is more dramatic; no multiplicity or symmetry-breaking is observed. Likewise when $H=10$. These results, along with those for the White-Metzner model, indicate that both shear thinning and the presence of a mechanism for rapid stress relaxation tend to simplify the dynamics of the flow. The phenomena may be related, as the effective Deborah number in the WhiteMetzner model decreases with increasing shear rate.

The above results indicate that the nonlinear dynamical behavior observed in the simple model Eqs. (11) is persistent with respect to many changes in the model. One level of modeling that has not been considered, however, is the choice of the basic model for the slip dynamics. Only a simple relaxation process has been used. More complicated models are available. For example, dependence of the slip on the normal stress could be included, as suggested by Hill et al. (1990). Furthermore, the relaxation-type model used here is not unique in incorporating the stress history into the slip velocity. For example, the variable-fluidity model of Yoshimura and Prud'homme (1988) also accomplishes this. 
At another level entirely, Hatzikiriakos and Kalogerakis (1994) have proposed a stochastic dynamical slip model based on network theory, but this model must be solved by stochastic simulation, a more complex and computationally demanding approach than is taken here.

\section{CONCLUSION}

The results presented here suggest that both viscoelasticity and a dynamic slip model are necessary to explain the instabilities and nonlinear dynamics of polymer melts in oscillatory shear. A simple model that incorporates these phenomena qualitatively reproduces experimental observations, including asymmetric periodic and nonperiodic responses. As a special case, this model reduces to the classical forced Duffing equation of nonlinear vibration theory. Furthermore, the model predicts the existence of a range of strain amplitudes over which two different stable responses are obtained, depending on initial conditions. The results are robust with respect to changes in both the constitutive model and the slip model. A validation of the slip model presented here would be the experimental observation of the predicted multiplicity. Experimentally this might be observed by performing experiments quasistatically increasing $\gamma_{0}$ and looking for a jump in the response of the shear stress or its phase relative to the forcing. Then the amplitude can be decreased and another jump back to the original response should be found. Beyond this, dynamic measurement of both slip velocity and wall stresses are needed to guide future theoretical and modeling studies. The success of a simple model in reproducing the observations suggests the possibility of using the large amplitude oscillatory shear experiment in tandem with nonlinear process identification techniques to develop empirical models for the dynamics of wall slip.

The importance of dynamic slip in oscillatory shear suggests that these dynamics may play a large role in the stability and dynamics of other flows where slip and unsteadiness are important. Not only linear stability limits and fully time-dependent flows, but also steady states, in which the flow at the wall is unsteady in the Lagrangian sense, will be influenced by the dynamics of wall slip.

\section{ACKNOWLEDGMENTS}

This work is partially supported by a Shell Faculty Fellowship. The author gratefully acknowledges helpful discussions and correspondence with S. G. Hatzikiriakos, M. Reimers and G. H. McKinley. The comments of M. M. Denn, J. M. Dealy, and A. J. Giacomin on this paper are greatly appreciated.

\section{APPENDIX}

In this appendix are compiled some rigorous results regarding the oscillatory shear behavior of isotropic simple liquids without slip, and of linear viscoelastic fluids with nonlinear algebraic slip models, $u_{s}=\phi(\tau)$. The main result, Theorem 3 , is that nonperiodic responses cannot occur in oscillatory shear of a general linear fluid with an algebraic slip law. By continuity, this result extends to weakly nonlinear viscoelasticity. This result is nontrivial, because even in the linear viscoelastic case, the nonlinearity of the slip model makes the full problem nonlinear. In the no-slip case, we show for a RivlinSawyers fluid that the response is periodic and symmetric: $\tau(t)=-\tau\left(t+\frac{1}{2}\right)$ (in dimensional variables, $\tau(t)=-\tau(t+T / 2)$, where $T$ is the oscillation period of the forcing function). We assume throughout that the stress and strain are uniform across the gap; under this assumption, the shear stress can be written in the nonlinear case as: 


$$
\tau(t)=-\int_{0}^{t} \psi\left(t-t^{\prime}, \gamma\left(t, t^{\prime}\right)^{2}\right) \gamma\left(t, t^{\prime}\right) d t^{\prime}
$$

Here, $\gamma\left(t, t^{\prime}\right)=\int_{t}^{t^{\prime}} \dot{\gamma}\left(t^{\prime \prime}\right) d t^{\prime \prime}$ and $\dot{\gamma}(t)=\dot{\gamma}_{n}(t)-2 \phi(\tau(t))$. For the general linear fluid model, we use

$$
\pi(t)=\int_{0}^{t} G\left(t-t^{\prime}\right) \dot{\gamma}\left(t^{\prime}\right) d t^{\prime}
$$

where $\psi\left(t-t^{\prime}, 0\right)=\partial G\left(t-t^{\prime}\right) / \partial t$. We assume that $\lim _{s \rightarrow \infty} G(s)=0$, and that

$$
\begin{gathered}
G(s)>0, \quad \frac{d G(s)}{d s}<0, \\
G(s) \frac{d^{2} G(s)}{d s^{2}}-\left(\frac{d G(s)}{d s}\right)^{2} \geqslant 0
\end{gathered}
$$

for all $s \geqslant 0$. These inequalities hold if $G(s)$ is positive and all its derivatives are monotone (Miller 1971). A superposition of decaying exponential functions, for example, satisfies these conditions. Finally, we require that $\dot{\gamma}_{n}(t)$ be bounded and continuous for all $t$, and that $\phi(\tau)$ be Lipschitz. Most, if not all, real polymer melts and experimental configurations will satisfy these conditions.

The proof of the periodicity result $\tau(t)=\tau(t+1)$ in the no-slip case is simple, essentially relying on the fading memory.

Thenrem 1. Consider a fluid whose stress is given by Eq. (14). If $\phi(\tau) \equiv 0$ and $\gamma\left(t, t^{\prime}\right)=\gamma\left(t+1, t^{\prime}+1\right)$, then $\lim _{t \rightarrow \infty}(\tau(t+1)-\tau(t))=0$.

Proof:

$$
\begin{aligned}
\gamma(t+1)-\gamma(t)= & -\int_{0}^{t+1} \psi\left(t+1-t^{\prime}, \gamma\left(t+1, t^{\prime}+1\right)^{2}\right) \gamma\left(t+1, t^{\prime}+1\right) d t^{\prime} \\
& +\int_{0}^{t} \psi\left(t-t^{\prime}, \gamma\left(t, t^{\prime}\right)^{2}\right) \gamma\left(t, t^{\prime}\right) d t^{\prime}
\end{aligned}
$$

Let $t^{\prime \prime}=t^{\prime}-1$ in the first integral, let $t^{\prime \prime}=t^{\prime}$ in the second and use the periodicity of $\gamma\left(t, t^{\prime}\right)$ to find that:

$$
\begin{aligned}
\pi(t+1)-\tau(t) & =-\int_{-1}^{t} \psi\left(t-t^{\prime \prime}, \mathcal{x}\left(t, t^{\prime \prime}\right)^{2}\right) x\left(t, t^{\prime \prime}\right) d t^{\prime \prime}+\int_{0}^{t} \psi\left(t-t^{\prime \prime}, \mathcal{x}\left(t, t^{\prime \prime}\right)^{2}\right) x\left(t, t^{\prime \prime}\right) d t^{\prime \prime} \\
& =-\int_{-1}^{0} \psi\left(t-t^{\prime \prime}, \chi\left(t, t^{\prime \prime}\right)^{2}\right) x\left(t, t^{\prime \prime}\right) d t^{\prime \prime} .
\end{aligned}
$$

Since $\lim _{s \rightarrow \infty} \psi\left(s, \gamma^{2}\right)=0$, this integral vanishes as $t \rightarrow \infty$, thus proving the result.

The symmetry result when $\gamma\left(t, t^{\prime}\right)=-\gamma\left(t+\frac{1}{2}, t^{\prime}+\frac{1}{2}\right)$ follows from a similar procedure:

Theorem 2. Consider a fluid whose stress is given by Eq. (14). If $\phi(\tau) \equiv 0$ and $\gamma\left(t, t^{\prime}\right)=-\gamma\left(t+\frac{1}{2}, t^{\prime}+\frac{1}{2}\right)$, then $\lim _{t \rightarrow \infty}\left(\tau(t)+\tau\left(t+\frac{1}{2}\right)\right)=0$.

Proof: 


$$
\begin{aligned}
\tau\left(t+\frac{1}{2}\right)+\gamma(t)= & -\int_{0}^{t+\frac{1}{2}} \psi\left(t+\frac{1}{2}-t^{\prime}, \gamma\left(t+\frac{1}{2}, t^{\prime}+\frac{1}{2}\right)^{2}\right) \gamma\left(t+\frac{1}{2}, t^{\prime}+\frac{1}{2}\right) d t^{\prime} \\
& \left.+\int_{0}^{t} \psi\left(t-t^{\prime}\right), \gamma\left(t, t^{\prime}\right)^{2}\right) \gamma\left(t, t^{\prime}\right) d t^{\prime}
\end{aligned}
$$

Let $t^{\prime \prime}=t^{\prime}-\frac{1}{2}$ in the first integral, let $t^{\prime \prime}=t^{\prime}$ in the second and use the fact that $\psi(s,-\gamma)=\psi(s, \gamma)$ :

$$
\begin{aligned}
\tau\left(t+\frac{1}{2}\right)+\tau(t) & =-\int_{-\frac{1}{2}}^{t} \psi\left(t-t^{\prime \prime}, \gamma\left(t, t^{\prime \prime}\right)^{2}\right) \gamma\left(t, t^{\prime \prime}\right) d t^{\prime \prime}+\int_{0}^{t} \psi\left(t-t^{\prime \prime}, \gamma\left(t, t^{\prime \prime}\right)^{2}\right) \gamma\left(t, t^{\prime \prime}\right) d t^{\prime \prime} \\
& =-\int_{-\frac{1}{2}}^{0} \psi\left(t-t^{\prime \prime}, \gamma\left(t, t^{\prime \prime}\right)^{2}\right) \gamma\left(t, t^{\prime \prime}\right) d t^{\prime \prime} .
\end{aligned}
$$

Again, the integral vanishes as $t \rightarrow \infty$, thus proving the result.

We turn now to the case of algebraic slip, considering only the case of linear viscoelasticity. In this case, we can prove that the stress response in oscillatory shear will always be periodic.

Theorem 3. Consider a fluid whose stress is given by Eq. $(A 2)$. If $\phi(0)=0$ and $\tau \phi(\tau) \geqslant 0$ for all $\tau$, and if $\dot{\gamma}_{n}(t)=\dot{\gamma}_{n}(t+1)$ for all $t$, then $\lim _{t \rightarrow x}(\tau(t+1)-\tau(t))=0$.

The proof of this theorem is nontrivial and will only be outlined here. The complete proof can be found in Miller (1971). Equation (A2) can be rearranged to yield:

$$
\tau(t)+2 \int_{0}^{t} G\left(t-t^{\prime}\right) \phi\left(\tau\left(t^{\prime}\right)\right) d t^{\prime}+g(t)=0
$$

where

$$
g(t)=-\int_{0}^{t} G\left(t-t^{\prime}\right) \dot{\gamma}_{n}\left(t^{\prime}\right) d t^{\prime} .
$$

Letting $y(t)=\tau(t)+g(t)$ yields

$$
y(t)=-2 \int_{0}^{t} G\left(t-t^{\prime}\right) \phi\left(y\left(t^{\prime}\right)-g\left(t^{\prime}\right)\right) d t^{\prime} .
$$

Theorem V.5.5 of Miller (1971) states that the unique solution of this equation as $t \rightarrow \infty$ is time-periodic with the same period as $\dot{\gamma}_{n}$. Technical details aside, the proof is based on the fact that the RHS of Eq. (A5) is a compact operator that maps asymptotically (as $t \rightarrow \infty$ ) periodic functions to asymptotically periodic functions. Therefore, by the Schauder fixed point theorem (cf. Zeidler 1986), the equation has a unique solution that is asymptotically time-periodic. Hence, for algebraic slip and linear viscoelasticity, the response must become periodic as $t \rightarrow \infty$. By continuity, this result is also valid for weakly nonlinear viscoelasticity-Eq. (A1) with small, but not infinitesimal, strain.

\section{References}

Adrian. D. W. and A. J. Giacomin, "The quasiperiodic nature of a polyurethane melt in oscillatory shear," J Rheol. 36, 1227-1243 (1992). (Often cited in the text as AG.)

Adrian. D. W. and A. J. Giacomin, "The transition to quasi-periodicity for molten plastics in large amplitude oscillatory shear." J. Eng. Mater. Tech. 116, 446-450 (1994). 
Atwood, B. T. and W. R. Schowalter, "Measurements of slip at the wall during flow of high-density polyethylene through a rectangular conduit," Rheol. Acta 28, 134-146 (1989).

Bird, R. B., R. C. Armstrong and O. Hassager, Dynamics of Polymeric Liquids, Fluid Mechanics, Vol. 1, 2nd ed. (Wiley, New York, 1987).

Denn, M. M., "Issues in viscoelastic fluid mechanics," Annu. Rev. Fluid Mech. 22, 13-34 (1990).

Denn, M. M., "Surface-induced effects in polymer melts," in Theoretical and Applied Rheology, edited by P. Moldenaers and R. Keunings (Elsevier, Amsterdam, 1992), pp. 45-49.

Doedel, E. J., "AUTO: a program for the automatic bifurcation analysis of autonomous systems," Congressus Numerantum 30, 265-284 (1981); Proceedings of the Tenth Manitoba Conference on Numerical Mathematics and Computation, University of Manitoba, Winnipeg, Canada, 1980 (unpublished).

Eckmann, J.-P. and D. Ruelle, "Fundamental limitations for estimating dimensions and Lyapunov exponents in dynamical systems," Physica D 56, 185-187 (1992).

Georgiou. G. C. and M. J. Crochet, "Compressible viscous flow in slits with slip at the wall," J. Rheol. 38, 639-654 (1994).

Grassberger, P. and I. Procaccia, "Characterization of strange attractors," Phys. Rev. Lett. 50, 346-349 (1983).

Guckenheimer, J. and P. Holmes, Nonlinear Oscillations, Dynamical Systems and Bifurcations of Vector Fields (Springer, New York, 1986).

Hatzikiriakos, S. G. and J. M. Dealy, "Wall slip of molten high density polyethylene. I. Sliding plate rheometer studies," J. Rheol. 35, 497-523 (1991). (Often cited in the text as HD.)

Hatzikiriakos, S. G. and J. M. Dealy, "Role of slip and fracture in the oscillating flow of HDPE in a capillary," J. Rheol. 36, 845-884 (1992).

Hatzikiriakos, S. G. and N. Kalogerakis, "A dynamic slip velocity model for molten polymers based un a network kinetic theory." Rheol. Acta 33, 38-47 (1994).

Hatzikiriakos, S. G., P. Hong, W. Ho and C. W. Stewart, "The effect of Teflon coatings in polyethylene capillary extrusion," J. Polym. Sci. 55, 595-603 (1995).

Hill, D. A., T. Hasegawa, and M. M. Denn. "On the apparent relation between adhesive failure and melt fracture," J. Rheol. 34, 891-918 (1990).

Jeyaseelan, R. J. and A. J. Giacomin, "Predicting polymer melt behavior near the inception of wall slip in oscillatory shear," J. Non-Newt. Fluid Mech. 53, 99-111 (1994).

Larson, R. G., "Instabilities in viscoelastic flows," Rheol. Acta 31, 213-263 (1992).

Leis, J. R. and M. A. Kramer, "ODESSA-an ordinary differential equation solver with explicit simultaneous sensitivity analysis," ACM Trans. Math. Software 14, 61-67 (1988).

Lim, F. J. and W. R. Schowalter. "Wall slip of narrow molecular weight distribution polyethylenes," J. Rheol. 33, 1359-1382 (1989).

Malkus, D. S., J. A. Nohel. and B. J. Plohr, "Analysis of new phenomena in shear flow of non-Newtonian fluids." SIAM J. Appl. Math. 51, 899-929 (1991).

Miller, R. K., Nonlinear Volterra Integral Equations (Benjamin, Menlo Park, CA, 1971 ).

Moon, F. C., Chaotic and Fractal Dynamics: An Introduction for Applied Scientists and Engineers (Wiley, New York, 19921

Pearson, J. R. A. and C. J. S. Petrie, "On melt flow instability of extruded polymers," in Polymer Systems: Deformation and Flow, edited by R. E. Wetton and R. H. Whorlow (Macmillan, London, 1968), pp. 163187.

Petrie, C. J. S. and M. M. Denn, "Instabilities in polymer processing," AIChE J. 22, 209-236 (1976).

Ramamurthy, A. V., "Wall slip in viscous fluids and influence of materials of construction," J. Rheol. 30, 337-357 (1986)

Renardy, M., "Short wave instabilities resulting from memory slip," J. Non-Newt. Fluid Mech. 35, 73-76 (1990).

Taylor, M. A., M. S. Jolly, and I. G. Kevrekidis, "Scigma," Technical report, Department of Chemical Engineering, Princeton University (1990).

Taylor, M. A. and I. G. Kevrekidis, "Interactive AUTo: a graphics interface for AUTO86," Technical report, Department of Chemical Engineering, Princeton University (1990).

Vinogradov, G. V., A. Ya. Malkin, Y. G. Yanovskii, E. K. Borisenkova, B. V. Yarlykov, and G. V. Berezhnaya, "Viscoelastic properties and flow of narrow polybutadienes and polyisoprenes," J. Polym. Sci. A 2, 1061 (1972).

Yoshimura, A. S. and R. K. Prud'homme, "Wall slip effects on dynamic oscillatory measurements," J. Rheol. 32, 575-584 (1988).

Zeidler, E., Nonlinear Functional Analysis and Applications I: Fixed-Point Theorems (Springer, New York, 1986). 